Article

\title{
Design and Testing of a Custom Melanoma Next Generation Sequencing Panel for Analysis of Circulating Tumor DNA
}

\author{
Russell J. Diefenbach 1,2 (D), Jenny H. Lee ${ }^{1,2}$, Alexander M. Menzies ${ }^{2,3,4}$, Matteo S. Carlino ${ }^{2,3,5}$, \\ Georgina V. Long ${ }^{2,3,4,6}$, Robyn P. M. Saw ${ }^{2,3,7}$, Julie R. Howle ${ }^{2,3,5}$, Andrew J. Spillane ${ }^{2,3,8}$, \\ Richard A. Scolyer 2,3,6,9 ${ }^{\mathbb{D}}$, Richard F. Kefford ${ }^{2,3,10}$ and Helen Rizos ${ }^{1,2, *}$
}

1 Department of Biomedical Sciences, Faculty of Medicine, Health and Human Sciences, Macquarie University, Sydney, NSW 2109, Australia; russell.diefenbach@mq.edu.au (R.J.D.); jenny.lee@mq.edu.au (J.H.L.)

2 Melanoma Institute Australia, The University of Sydney, Sydney, NSW 2065, Australia; alexander.menzies@sydney.edu.au (A.M.M.); matteo.carlino@sydney.edu.au (M.S.C.); georgina.long@sydney.edu.au (G.V.L.); robyn.saw@melanoma.org.au (R.P.M.S.); julie.howle@sydney.edu.au (J.R.H.); andrew.spillane@sydney.edu.au (A.J.S.); richard.scolyer@health.nsw.gov.au (R.A.S.); richard.kefford@mq.edu.au (R.F.K.)

3 Sydney Medical School, The University of Sydney, Sydney, NSW 2006, Australia

4 Department of Medical Oncology, Northern Sydney Cancer Centre, Royal North Shore Hospital, Sydney, NSW 2065, Australia

5 Crown Princess Mary Cancer Centre, Westmead and Blacktown Hospitals, Sydney, NSW 2145, Australia

6 Charles Perkins Centre, The University of Sydney, Sydney, NSW 2006, Australia

7 Department of Melanoma and Surgical Oncology, Royal Prince Alfred Hospital, Sydney, NSW 2050, Australia

8 Breast and Melanoma Surgery Department, Division of Surgery, Royal North Shore Hospital, Sydney, NSW 2065, Australia

9 Department of Tissue Pathology and Diagnostic Oncology, Royal Prince Alfred Hospital and New South Wales Health Pathology, Sydney, NSW 2050, Australia

10 Department of Clinical Medicine, Faculty of Medicine, Health and Human Sciences, Macquarie University, Sydney, NSW 2109, Australia

* Correspondence: helen.rizos@mq.edu.au; Tel.: +61-298-502-762

Received: 30 June 2020; Accepted: 7 August 2020; Published: 10 August 2020

check for updates

\begin{abstract}
Detection of melanoma-associated mutations using circulating tumor DNA (ctDNA) from plasma is a potential alternative to using genomic DNA from invasive tissue biopsies. In this study, we developed a custom melanoma next-generation sequencing (NGS) panel which includes 123 amplicons in 30 genes covering driver and targetable mutations and alterations associated with treatment resistance. Analysis of a cohort of 74 stage III and IV treatment-naïve melanoma patients revealed that sensitivity of ctDNA detection was influenced by the amount of circulating-free DNA (cfDNA) input and stage of melanoma. At the recommended cfDNA input quantity of $20 \mathrm{ng}$ (available in $28 / 74$ patients), at least one cancer-associated mutation was detected in the ctDNA of $84 \%$ of stage IV patients and $47 \%$ of stage III patients with a limit of detection for mutant allele frequency (MAF) of $0.2 \%$. This custom melanoma panel showed significant correlation with droplet digital PCR (ddPCR) and provided a more comprehensive melanoma mutation profile. Our custom panel could be further optimized by replacing amplicons spanning the TERT promoter, which did not perform well due to the high GC content. To increase the detection rate to $90 \%$ of stage IV melanoma and decrease the sensitivity to $0.1 \% \mathrm{MAF}$, we recommend increasing the volume of plasma to $8 \mathrm{~mL}$ to achieve minimal recommended cfDNA input and the refinement of poorly performing amplicons. Our panel can also be expanded to include new targetable and treatment resistance mutations to improve the tracking of treatment response and resistance in melanoma patients treated with systemic drug therapies.
\end{abstract}


Keywords: Melanoma; circulating tumor DNA; targeted sequencing; custom panel

\section{Introduction}

The analysis of circulating tumor DNA (ctDNA) is progressively being integrated into routine clinical care to monitor cancer progression, response to therapy, emergence of resistance and to direct therapy [1-6]. In melanoma, longitudinal ctDNA assessment predicts overall survival of stage IV melanoma patients treated with either BRAF and MEK inhibitors or immunotherapy [7-11] and the survival of patients with high-risk stage III resected melanoma [12-15]. ctDNA can also monitor the appearance of treatment-resistant melanoma subclones [7], tumor heterogeneity [16], metabolic tumor burden [17] and differentiate "true progression" from "pseudoprogression" in melanoma patients treated with immunotherapy [18].

Melanoma ctDNA is often detected using single gene assays that monitor a driver mutation that has been previously identified in patient-matched cancer tissue $[7,8,12,15,19]$. Although next generation sequencing (NGS) can provide an unbiased and comprehensive mutation profile of ctDNA, it is technically challenging due to the limited quantities of highly-fragmented ctDNA [20]. Furthermore, with increased genomic coverage and sequencing error rates in the order of $0.1-1 \%$, whole-exome NGS of ctDNA does not provide the limit of detection (LOD) required to accurately identify low frequency mutations $(<1 \%)$, which may occur in pre-existing or emerging treatment resistant subclones $[16,21]$. The use of customized gene mutation panels in NGS of ctDNA can produce significantly lower levels of mutation detection [22], and these panels typically monitor common melanoma driver mutations in $B R A F, N R A S$ and KIT, along with mutations in tumor suppressor genes, such as TP53. However, such gene panels do not include many established mutations associated with treatment resistance and do not allow for the discovery and tracking of novel resistance mutations [22]. A commercially-available targeted melanoma panel (UltraSEEK) for ctDNA, which covers 61 mutations in 13 genes with a LOD of $0.1 \%$, has been developed for the study of melanoma disease progression and resistance to systemic treatments [23-25]. The UltraSEEK panel is based on mutation detection by mass spectrometry, and, although it showed similar sensitivity to ddPCR in Stage IV melanoma known to carry mutations in plasma, the concordance in the mutations detected was only $88 \%$ between these two platforms [24]. Furthermore, in one study the UltraSEEK detection rate for stage IV melanoma was only $66.7 \%$ [25]. To date, only one study has employed a targeted custom melanoma NGS panel (950 amplicons over 30 genes) to analyze ctDNA and matched tissue from a cohort $(n=24)$ of stage IV melanoma patients [26]. In this small study, a confirmed driver mutation was identified in $70 \%$ of matching plasma samples.

In this study, we developed a melanoma NGS panel for ctDNA analysis based on 123 amplicons covering 30 genes with a predicted detection rate of $90 \%$ for cutaneous melanoma and $95 \%$ for uveal melanoma $[27,28]$. The performance of this melanoma mutation panel was evaluated in 74 treatment-naïve stage III and IV melanoma patients to identify whether stage III and IV melanoma could be monitored using a simple blood test.

\section{Results}

\subsection{Cohort and Sample Characteristics}

In total, 91 consecutive patients were recruited between April 2018 and December 2019. Seventeen patients were excluded from analysis as they were subsequently confirmed to have a non-melanoma malignancy $(n=3)$ or the liquid biopsy sample was taken after surgical resection $(n=14)$. Of the evaluable 74 treatment-naïve melanoma patients, 36 (49\%) patients had stage III and 38 (51\%) had stage IV melanoma (Table 1). The median age of the cohort was 65 years (range 23-89), the majority were male $(n=54,73 \%)$ and all but one patient had cutaneous melanoma (uveal melanoma, $n=1)$. Lymph node metastasis were present in 27/36 (75\%) stage III patients. Of these, 26/27 (96\%) patients 
had clinically-detectable lymph node metastasis, whereas 1/27 (4\%) patients had subclinical lymph node metastases at time of liquid biopsy. The median diameter of the largest lymph node metastases was $28 \mathrm{~mm}$ (range 2-81 mm). The remaining nine patients had in-transit metastases only. Of the patients with stage IV disease, 5 patients had M1a, 7 had M1b, 16 had M1c and 10 had M1d disease (with concurrent extracranial metastases in 9/10 patients).

Table 1. Summary of patient cohort.

\begin{tabular}{|c|c|c|}
\hline Characteristics & $\begin{array}{l}\text { Stage III } \\
(n=36)\end{array}$ & $\begin{array}{c}\text { Stage IV } \\
(n=38)\end{array}$ \\
\hline \multicolumn{3}{|l|}{ Age (years) } \\
\hline Median (range) & $66(32-89)$ & $61(23-88)$ \\
\hline \multicolumn{3}{|l|}{ Sex, n (\%) } \\
\hline Male & $26(72)$ & $28(74)$ \\
\hline Female & $10(28)$ & $10(26)$ \\
\hline \multicolumn{3}{|l|}{ Disease distribution, $\mathrm{n}(\%)$} \\
\hline LN metastases & $27(75)$ & \\
\hline IT disease only & $9(25)$ & \\
\hline \multicolumn{3}{|l|}{ AJCC M stage, n (\%) } \\
\hline M1a & & $5(13)$ \\
\hline M1b & & $7(18)$ \\
\hline M1c & & $16(42)$ \\
\hline M1d & & $10(26)$ \\
\hline \multicolumn{3}{|l|}{ Tissue mutation profile, $\mathrm{n}(\%)$} \\
\hline$B R A F$ & $11(31)$ & $15(39)$ \\
\hline NRAS & $8(22)$ & $3(8)$ \\
\hline$B R A F \mathrm{WT}^{1}$ & $7(19)$ & $3(8)$ \\
\hline$B R A F / N R A S / K I T \mathrm{WT}^{2}$ & $5(14)$ & $15(39)$ \\
\hline Not performed & $5(14)$ & $2(5)$ \\
\hline cfDNA, $\mathrm{n}(\%)$ & & \\
\hline Threshold for NGS $(20 \mathrm{ng} / 16.5 \mu \mathrm{L})$ & $9(25)$ & $19(50)$ \\
\hline
\end{tabular}

${ }^{1}$ VE1 immunohistochemistry negative for BRAF V600E. ${ }^{2}$ No mutation found on molecular testing. Abbreviations: NGS, next generation sequencing; LN, lymph node; IT, in-transit.

At least $4 \mathrm{~mL}$ of plasma was available for 72/74 (97\%) patients, and the total recovered quantity of cfDNA ranged from 7.2-266.8 ng (median $22.75 \mathrm{ng}$ ). cfDNA quantity was significantly higher in patients with stage IV than stage III disease; median cfDNA was $28.75 \mathrm{ng}$ versus $18.45 \mathrm{ng}$, respectively (Figure 1). Using a cfDNA threshold for NGS of $20 \mathrm{ng}$ in $16.5 \mu \mathrm{L}$ (i.e., $1.21 \mathrm{ng} / \mu \mathrm{L}$ as recommended in the Ion Ampliseq HD library kit user guide), 9/36 (25\%) stage III and 19/38 (50\%) stage IV patients met this cfDNA threshold (Table 1).

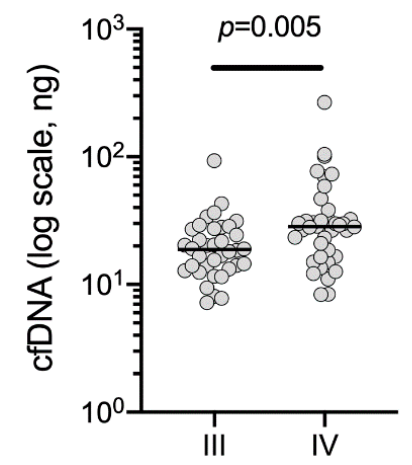

Figure 1. Total yield of cfDNA per patient for stage III vs. stage IV melanoma patients. Mann-Whitney, unpaired, nonparametric $t$-test was performed. 


\subsection{Evaluation of the Performance of the Custom Melanoma Panel}

The performance of the melanoma panel (Table S1) was initially evaluated based on amplicon reading depth, with an expected $\sim 120,000 \mathrm{X}$ median read depth per amplicon according to the capacity of the Ion 550 sequencing chip with seven samples run per chip with the manufacturer's recommended $20 \mathrm{ng}$ cfDNA input (Figure 2). As expected, reading depth was significantly lower (average 100,000X) when input cfDNA was less than $20 \mathrm{ng}$, and was also diminished when input cfDNA was increased to $>20 \mathrm{ng}$ (Figure 2). This was particularly evident when we compared patient-matched amounts of 20 and $30 \mathrm{ng}$ cfDNA from two melanoma patients and one patient with a non-melanoma malignancy. A pairwise comparison showed a significant decrease in total amplicon reads when cfDNA template was increased to $30 \mathrm{ng}$ (Figure 3A). In contrast, there was a significant increase in the number of molecular tag families (Figure 3B), and this was associated with a significant decrease in LOD (Figure 3C) with no detectable changes in MAF (Figure 3D).

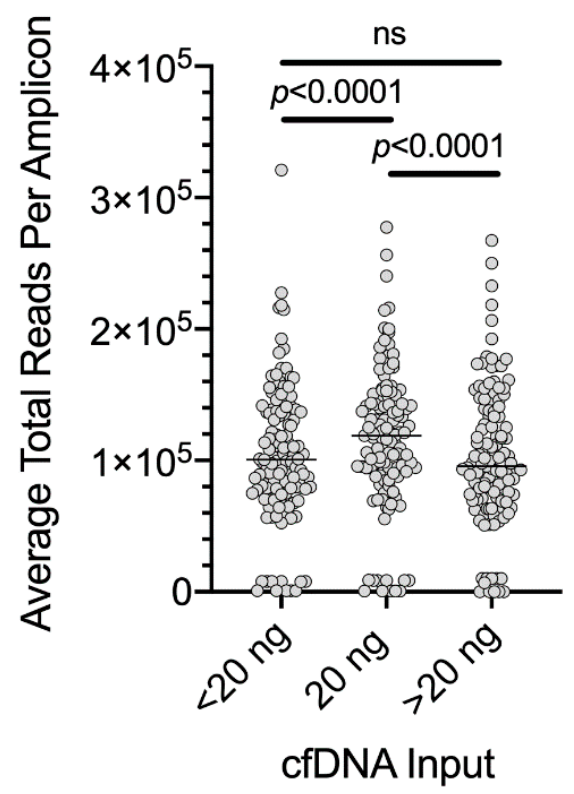

Figure 2. Average total reads obtained for each amplicon in the custom melanoma panel. The input template cfDNA was $<20 \mathrm{ng}$ ( $n=40$ samples, range of 3.6-19.1 ng), $20 \mathrm{ng}$ ( $n=21$ samples, range of $19.5-20.5 \mathrm{ng}$ ) or $>20 \mathrm{ng}$ ( $n=8$ samples, range of $21-42 \mathrm{ng}$ ). In each case, seven NGS sample libraries were run on an Ion 550 sequencing chip using the Ion Ampliseq HD workflow. Reading depth data for each amplicon for each patient are shown in Table S2. One-way ANOVA with Tukey's multiple comparison test was performed. ns, not significant. 


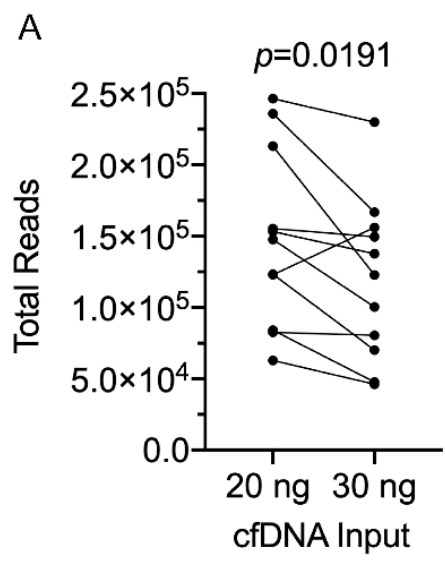

C

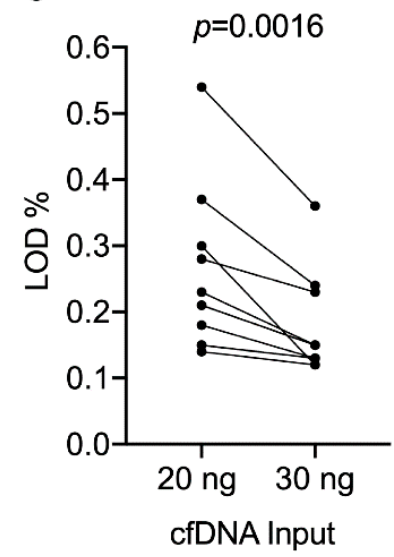

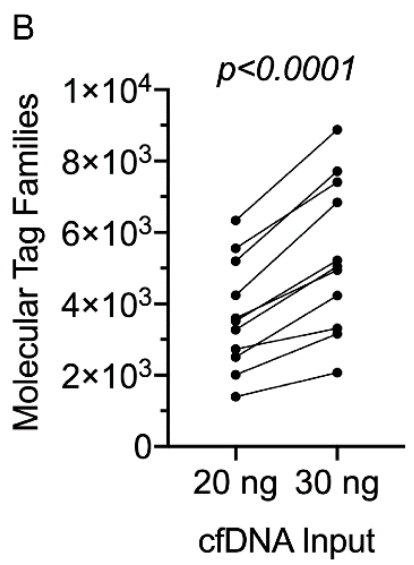

D

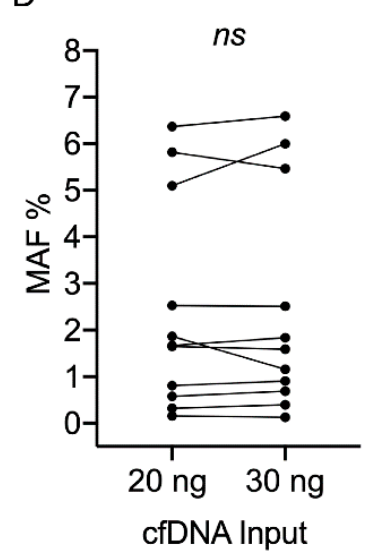

Figure 3. Relationship between sequencing performance, variant detection and cfDNA template input. (A-D) Pairwise comparison of mutations detected in matched patient samples (refer to Table S3) using either 20 or 30 ng cfDNA template input: (A) total reads; (B) molecular tag families (defined in materials and methods) per individual amplicon; (C) limit of detection (LOD); and (D) mutant allele frequency (MAF). Parametric paired $t$-tests were performed. ns, not significant.

Regardless of the median amplicon sequencing reading depth or coverage (number of unique reads) achieved for the melanoma panel, the relative performance of individual amplicons remained consistent with similar relative read depth regardless of the amount of input template cfDNA (Figure S1). Several amplicons, including the amplicon spanning EIF1AX amino acids 6-13 and the TERT promoter amplicons showed consistently lower reading depths relative to other amplicons (Figure S1). As a result, no EF1AX or TERT promoter mutations were detected in our patient cohort. Mutation calls could not be made for the TERT promoter amplicons because no reverse sequencing reads were obtained (data not shown).

The LOD in our panel was analyzed by spiking patient samples with NRAS A59T and Q61K mutations at the MAFs of $1.3 \%, 0.26 \%$ and $0.13 \%$ (Horizon standards). The melanoma panel consistently detected both NRAS mutations at $1.3 \%$ and $0.26 \%$ frequency but did not accurately detect the NRAS mutations at $0.13 \%$ MAF (Table S4). This indicates the LOD for our custom panel was approximately $0.2 \%$.

\subsection{Identification of Melanoma Mutations Using the Custom Melanoma Panel}

Melanoma mutations, either driver (BRAF, NRAS, NF1, GNAQ; identified in $n=29$ patients) or other cancer-associated mutation (identified in $n=16$ patients; defined in Table S5), were identified in $45 / 74(61 \%)$ melanoma patients using our custom melanoma panel, 17/36 (47\%) for stage III and 28/38 $(74 \%)$ for stage IV (Figure $4 \mathrm{~A})$. When tumor tissue mutation testing was available, a matched ctDNA and tumor driver mutation was confirmed in 19/19 patients (Figure 4A). ctDNA melanoma mutation 
detectability was also stratified according to disease distribution and stage. For stage III patients, ctDNA melanoma mutation detectability was 14/27 (52\%) for patients with lymph node metastasis and $3 / 9(33 \%)$ for patients with in-transit metastases only, without lymph node metastases (Figure $4 \mathrm{~A}$ ). For stage IV patients, detectability was 3/5 (60\%) for M1a, 6/7 (86\%) for M1b, 12/16 (75\%) for M1c and $7 / 10(70 \%)$ for M1d melanoma (Figure 4A). Increasing input cfDNA increased mutation detection in stage IV patients but not in stage III patients. In particular, whereas only $12 / 19(63 \%)$ stage IV patients had detectable mutations with input cfDNA $<20 \mathrm{ng}$, this increased to $16 / 19(84 \%)$ mutations detected with input cfDNA $\geq 20 \mathrm{ng}$ (maximum of $30 \mathrm{ng}$ ) (Figure 4B).
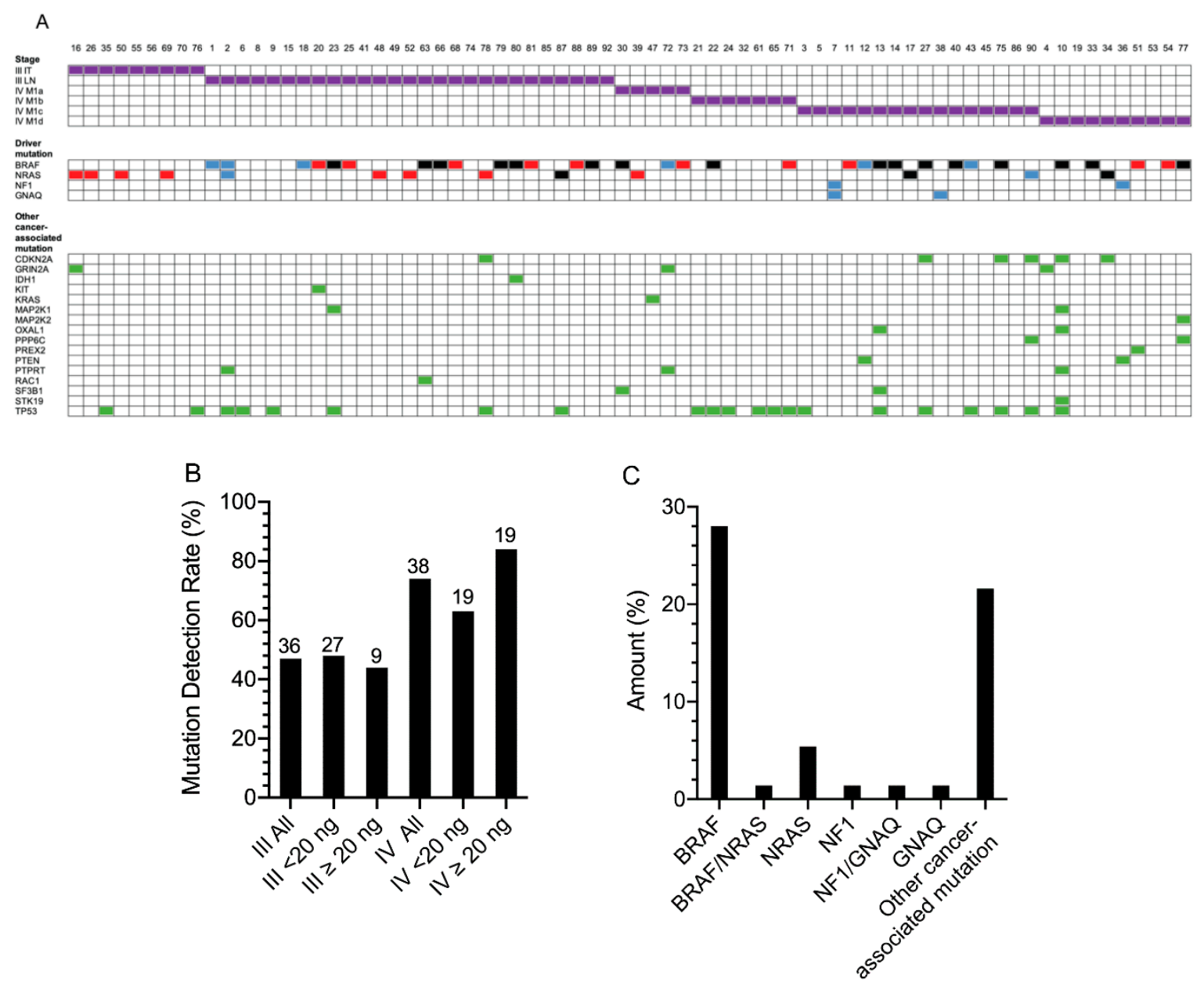

Figure 4. Summary of the melanoma mutation profile and rate of detection based on the custom melanoma panel. (A) Comparison of melanoma stage (purple boxes), driver mutation detected by tissue biopsy (red boxes), driver mutation detected by liquid biopsy (blue boxes), driver mutation detected by both liquid and tissue biopsy (black boxes) and other cancer-associated mutations (green boxes) identified across the cohort of 74 melanoma patients. Numbers represent patient sample number. IT, in-transit; LN, lymph node. For details on specific gene mutations and complete NGS data, refer to Tables S5 and S6. (B) Comparison of mutation detection rate for melanoma mutations based on the level of cfDNA used for NGS. Total sample number is shown above each histogram. For details on stage, cfDNA input and melanoma driver or other cancer-associated mutation detected, refer to Table S5. (C) Distribution of melanoma driver mutation (BRAF, GNAQ, NF1 and NRAS) or other cancer-associated mutation (only including those which lacked a BRAF, GNAQ, NF1 or NRAS driver mutation). Amount indicates percent of total melanoma cohort $(n=74)$.

We were particularly interested in the 10 stage IV patients who did not have a detectable driver or other cancer-associated mutation in the ctDNA using our custom melanoma panel. Nine of these patients had driver mutation data derived from the tumor specimen: 5/9 patients had BRAF/NRAS/KIT 
wild type melanoma, whereas 4/9 patients had BRAF or NRAS driver mutations identified in the tumor. In particular, NRAS Q61L and BRAF V600E mutations were identified in two patients with M1a disease, while BRAF V600E and BRAF D594N mutations were found in one patient each with M1c and M1d disease, respectively. Both patients with M1c and M1d disease had very low volume disease: the M1c patient had 6-mm lung and 23-mm peritoneal metastases and the M1d patient had a brain metastasis and a subcentimeter solitary lung metastasis.

We identified BRAF mutations in 21/74 (28\%) patients, NRAS mutations in 4/74 (5.4\%) patients, NF1 mutations in 1/74 (1.4\%) patients and GNAQ mutations in 1/74 (uveal melanoma) (1.4\%) patients, as well as BRAF/NRAS $(1 / 74 ; 1.4 \%)$ and GNAQ/NF1 $(1 / 74 ; 1.4 \%)$ double mutations (Figure 4 C). Other cancer-associated mutations, including TP53, CDKN2A and PTEN alterations, were also identified in another 16/74 (22\%) patients (Figure 4C). Other mutations were identified in 20 patients who did not have a detectable melanoma driver or other cancer-associated mutation (Table S5). The majority of these mutations were detected at low MAFs and were below the LOD and thus would require further validation. Only $4 / 20$ patients had other ctDNA-associated mutations detected at a MAF of $>0.2 \%$ (GRIN2A P1171L, RAC1 A27P, STK19 T80N and TP53 V173M).

The concordance between tissue and ctDNA driver mutations was performed in 36 of the 38 stage IV patients who had undergone tissue mutation analysis (three patients had VE1 immunohistochemistry only) (Table S7). In 18/36 patients, a melanoma driver mutation was detected in the tissue specimen and a matching ctDNA mutation was detected in 12 of these patients (66.7\%) (Figure 5). In Patients 13 and 33, an additional rare BRAF driver mutation was identified in the ctDNA sample (Table S7). In the 18 patients with no driver mutation detected in the melanoma tissue biopsy, six patients had a driver mutation identified in the ctDNA (Figure 5), including three patients with rare BRAF kinase domain mutations (BRAF G466A, BRAF G469A and BRAF T599dup) and one patient with a predominant NF1 R1241* nonsense mutation (MAF 27.65\%) and a low frequency GNAQ R183Q mutation (MAF 0.71\%) (Table S7). Cancer-associated mutations were also identified in the ctDNA of 9/36 patients (Table S7), and, although these were not validated in this study, it is worth noting that six of these nine patients $(67 \%)$ had no driver mutation identified in the tissue biopsy.

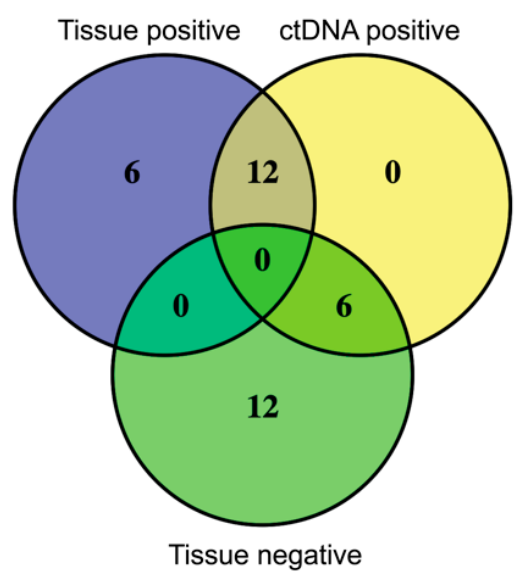

Figure 5. Concordance between tissue and ctDNA driver mutations for stage IV melanoma patients $(n=36)$. Values shown represent number of patients.

\subsection{Validation of Custom Melanoma Panel}

We initially analyzed the performance of our custom melanoma panel by validating driver mutations identified in the ctDNA of 13 selected patients. These patients were chosen based on the availability of ddPCR probes and sufficient cfDNA template. Using ddPCR, we validated all 13 driver mutations and confirmed there was a significant correlation in the MAFs generated by ddPCR and our custom NGS assay (Figure 6A). 


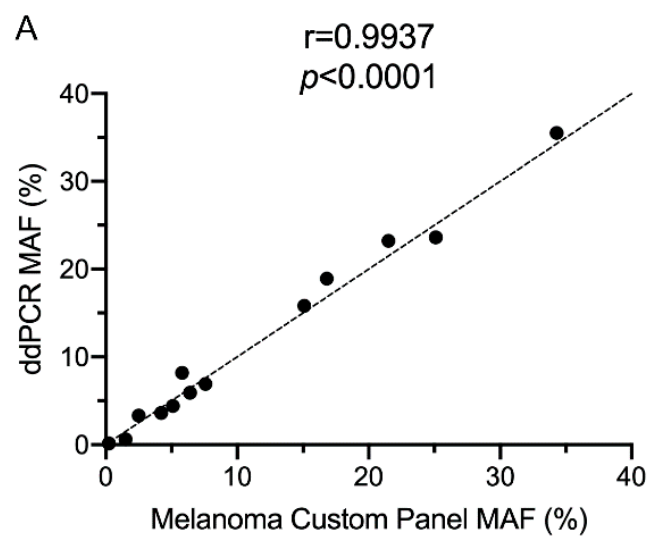

B

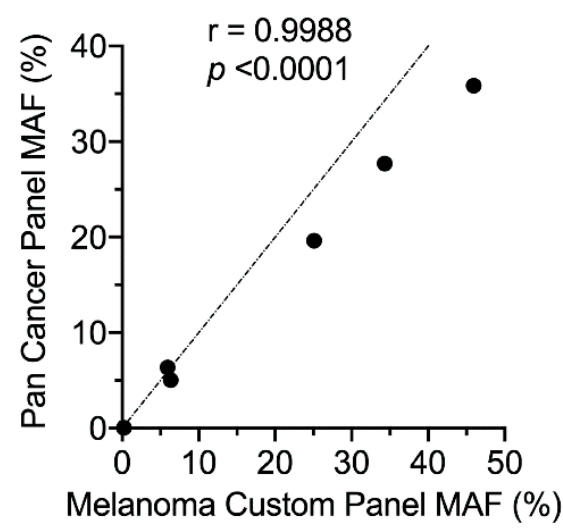

C

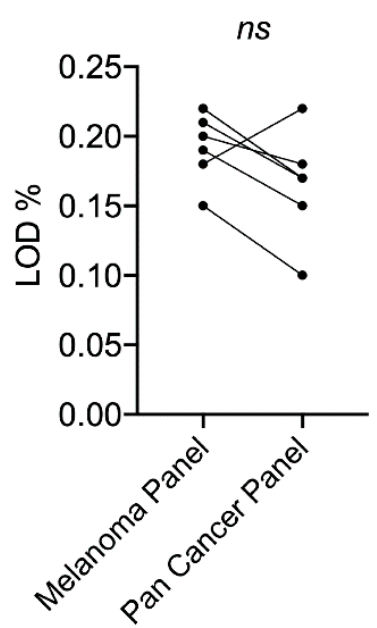

Figure 6. Validation of the custom melanoma panel. (A) Correlation of mutant allele frequency (MAF) determined by the melanoma custom panel versus ddPCR analysis. (B,C) Comparison of custom melanoma and Oncomine pan cancer targeted NGS panels. (B) Correlation of MAF based on mutations detected by both panels. (C) Pairwise comparisons of limit of detection (LOD) for matched mutations detected using both assays. For patient samples tested along with NGS and ddPCR data refer to Tables S8 and S9. Pearson correlation coefficient analysis and parametric paired $t$-test were performed.

Next we compared the performance of our custom melanoma panel with the 52-gene Thermofisher Oncomine pan cancer panel. For this analysis, five patients from our melanoma cohort plus three patients from the non-melanoma patients were selected based on ctDNA availability. There was significant correlation in the MAF for each identified mutation between the two cancer panels (Figure 6B). We also observed that the LOD of driver and cancer-associated mutations was not significantly different between the two cancer panels, although the custom panel did not reach the specified value of $0.1 \%$ (Figure 6C).

\subsection{TERT Promoter Mutations}

We also took the opportunity to assess TERT promoter mutations using ddPCR in 10 patients. The $-124 \mathrm{C}>\mathrm{T}$ and $-146 \mathrm{C}>\mathrm{T}[29,30]$ mutations were not detected using our custom melanoma panel and $-124 \mathrm{C}>\mathrm{T}$ was identified using ddPCR in patients 34 and 56 at a MAF of $0.8 \%$ and $0.15 \%$, respectively (Table S10). Patient 34 also had a NRAS Q61K mutation, confirmed by both tissue and ctDNA NGS. The manual evaluation of the sequencing Bam files from our custom panel confirmed the presence of the $-124 \mathrm{C}>\mathrm{T}$ TERT mutation in patients 34 and 56 with MAFs of $1.1 \%$ and $0.17 \%$, respectively. A TERT promoter mutation, $-146 \mathrm{C}>\mathrm{T}$, was found in patient 88 at a MAF of $0.33 \%$ using 
ddPCR, although this was with only one positive droplet in the mutant channel. This mutation was not detected in the NGS sequencing Bam file generated using the custom melanoma panel.

\section{Discussion}

There are now many commercially available tumor-specific NGS panels for liquid biopsy, for cancers such as lung, breast and colorectal. In addition, there are several pan-cancer liquid biopsy NGS panels which provide the flexibility of covering several cancers but at a lower depth of coverage for known tumor-specific driver mutations [22]. To the best of our knowledge, there is only one cutaneous melanoma specific panel for liquid biopsy on the market $[23,24]$ which includes only 61 variants across 16 genes. In this study, we developed a broader melanoma-specific panel for liquid biopsy with a higher coverage of mutations for both cutaneous and uveal melanoma.

We developed and tested a custom melanoma NGS panel, based on Ampliseq HD, on a cohort of 74 melanoma patients which had a theoretical coverage of $90 \%$ of cutaneous melanoma and $95 \%$ of uveal melanoma $[27,28]$. With an optimal cfDNA input of $20 \mathrm{ng}$, we reached a $74 \%$ detection rate, based on detection of driver or cancer-associated mutation, of metastatic disease (stage III and IV patients), which increased to an $84 \%$ detection rate in stage IV melanoma patients.

To improve the performance of this panel, we plan to remove or improve underperforming amplicons which take up sequencing reads, such as those covering TERT promoter (discussed below), $D D X 3 X$ and EIF1AX. Both DDX $3 X$ and EIF1AX mutations account for $10 \%$ of melanomas and they generally co-occur with NRAS mutations [27,28]. Although their removal from the panel should have no significant impact on the overall detection rate of mutations in ctDNA, these mutations may have prognostic value [31-33] and improving amplicon design will be explored. Another option to improve the detection rate of our panel would be to increase the mutation analysis coverage of NF1. Based on ctDNA, just 3\% of this cohort had NF1 mutations, which is significantly lower than the TCGA value of $10 \%$ for cutaneous melanoma [27,28]. Given NF1 exons span $8520 \mathrm{bp}$ and sequencing amplicons are typically less than $100 \mathrm{bp}$, it would not be feasible to cover the complete NF1 gene, but the select inclusion of 10 additional amplicons would cover the majority of NF1 mutations identified in $B R A F / N R A S$ wild-type melanoma patients and should increase our coverage to $\sim 90 \%[27,28]$.

The MAF of a BRAF G469A mutation based on ctDNA using our custom panel was found to be $<0.2 \%$ in two stage IV melanoma patients. These same mutations were not detected in the matching tissue biopsy. We presume that these mutations are subclonal and therefore may not be represented (or exist at very low frequency) in the matching tissue biopsy.

Based on MAF, our custom melanoma panel showed a correlation of 0.994 when compared to ddPCR analysis and 0.9988 when compared to analysis with an off-the-shelf pan cancer NGS panel. We found that our custom panel did not reach the expected LOD of $0.1 \%$ (based on the sensitivity of ddPCR and off-the-shelf panels [22]). Typically, for the Ampliseq HD NGS workflow used with our custom panel, $\geq 3000$ molecular tag families/amplicon are required to achieve a LOD of $0.1 \%$ (personal communication from Thermofisher). The number of molecular tag families present across the NRAS amplicon, present in Horizon reference standard, was found to be in the range 1400-1800 for our custom melanoma panel. This would explain why for our custom melanoma panel, when using the optimum $20 \mathrm{ng}$ of template, we only achieved a LOD of $\sim 0.2 \%$ when testing the Horizon reference standards.

Many studies using NGS panels do not report the LOD relative to MAF. In our study, we comprehensively reported both values to emphasize the care required when calling mutations with low MAF $(<0.5 \%)$ which may be less than the LOD. We found that increasing cfDNA directly correlated with increasing molecular tag families in our sequencing libraries which inversely correlated with the LOD. Unexpectedly, cfDNA input above $20 \mathrm{ng}$ produced lower reading depths, although these data should be interpreted with caution as they were derived from a small number of sequencing runs. Collectively, our data indicate that increased plasma volumes would be expected to lower the LOD and is worth considering, especially as there is a linear recovery of cfDNA up to $17.5 \mathrm{~mL}$ of 
input plasma with the extraction kit used here [34]. Given that $50 \%$ of stage IV patients had less than the recommended cfDNA input of $20 \mathrm{ng}$ (we observed an increase in detection rate from $63 \%(<20$ ng cfDNA) to $84 \%$ ( $\geq 20$ ng cfDNA) for stage IV) increasing the amount of available cfDNA, through processing $8 \mathrm{~mL}$ of plasma, would also increase our detection rate in stage IV melanoma.

We found that $28 \%(21 / 74)$ of our patient cohort had TP53 mutations. Some of these p53 mutations have been shown to be a result of clonal hematopoiesis. This is a process whereby expansion of blood cell clones occurs due to advantageous somatic mutations, which often accumulate with age, and may contribute to hematological malignancies [35-38]. Having a broad NGS panel, that can simultaneously detect multiple low frequency variants becomes critical in defining the significance of circulating mutations.

The TERT promoter mutations $-124 \mathrm{C}>\mathrm{T}$ and $-146 \mathrm{C}>\mathrm{T}$ tend to be mutually exclusive and are reported to account for anywhere from 32\% (based on NGS analysis of tissue [26]) to 68-78\% (based on ddPCR analysis of tissue $[39,40]$ ) of all melanomas. As already highlighted, TERT promoter amplicons did not perform well in our custom melanoma panel, presumably due to the high GC content of around $80 \%$, and we did not detect any TERT promoter mutations in our cohort. A similar observation has been reported using a custom melanoma NGS panel analysis of plasma ctDNA from stage IV melanoma patients [26]. The Guardant360 pan cancer NGS panel (covers 73 genes) developed by Guardant Health has successfully detected TERT promoter mutations in ctDNA from a number of cancers, although melanoma was not included [41,42]. The methodology for achieving successful sequencing of the TERT promoter was not disclosed in these published studies. Importantly, TERT promoter mutations are detectable by ddPCR and the assay sensitivity can be enhanced by incorporation of 7-deaza-dGTP $[26,39,43]$. We therefore also incorporated 7-deaza-dGTP in our ddPCR assay to analyze ctDNA from 10 of our patient cohort and found $30 \%$ were positive for TERT promoter mutations. The MAF of these TERT promoter mutations were low (in the range $0.15-0.8 \%$ ) compared to values of $2-20 \%$ previously reported for a cohort of stage IV melanoma patients [26].

Ideally, detection of TERT promoter mutations needs to be included in any future workflow to maximize detection of melanoma based on ctDNA. Given the difficulty encountered with sequencing of the TERT promoter using NGS, an alternative custom melanoma panel based on ddPCR could be developed. This panel could include multiplex screening with primer/probes for detection of BRAF V600E/K/R, NRAS G12A/C/D/S/V, NRAS G13D/R/V, NRAS Q61H/K/L/R along with pooled primer/probes for TERT promoter mutations $-124 \mathrm{C}>\mathrm{T}$ and $-146 \mathrm{C}>\mathrm{T}$. This concept of following $B R A F, N R A S$ and TERT in the context of melanoma in a multiplex assay has been explored in a recent study [40]. This custom ddPCR panel would have a predicted coverage of $\sim 80 \%$ of cutaneous melanomas based on the skin cutaneous melanoma TCGA dataset which gives a BRAF/NRAS coverage of $\sim 70 \%[27,28]$ and the fact that TERT promoter mutations are found in around $\sim 29 \%$ of BRAF wild-type melanoma patients [39]. Analysis of NRAS and BRAF mutations in stage IV melanoma patients by single reaction ddPCR has shown an overall detection rate of $73 \%$ [7]. Such a single multiplex ddPCR reaction is feasible given the recent release of new digital PCR platforms from both Bio-Rad and Qiagen with multiplexing capabilities due to the incorporation of four color channels. Such a ddPCR panel, with lower cost, turnaround time of 1 day and requirement for less cfDNA $(<10$ $\mathrm{ng}$ ) than the NGS assay ( $>20 \mathrm{ng}$ ), could be used as a first pass for detection of melanoma mutations using ctDNA. Given the ddPCR assay requires less cfDNA template it would also therefore require less plasma (typically $2 \mathrm{~mL}$ ). Those melanoma patients who fail detection with the custom ddPCR panel could then be analyzed using our optimized ( $~ 90 \%$ coverage) custom melanoma NGS panel. The NGS assay would require $4-8 \mathrm{~mL}$ of plasma to obtain sufficient cfDNA template with the precise volume of plasma determined by the initial cfDNA extraction used for ddPCR. 


\section{Materials and Methods}

\subsection{Human Melanoma Samples}

The fresh-frozen tissue and blood samples used in the current study were obtained from the Melanoma Institute Australia biospecimen bank with written informed patient consent and institutional review board approval (Sydney Local Health District Human Research Ethics Committee, Protocol No. X15-0454 and HREC/11/RPAH/444). The Oncofocus panel (Agena Bioscience, San Diego, CA, USA) was used for detection of melanoma-associated BRAF, NRAS, KRAS and KIT variants in paired tissue samples $[44,45]$. Immunohistochemistry to detect BRAF V600E using VE1 monoclonal antibody (Abcam, Cambridge, UK) was performed as previously described [46].

Blood $(10 \mathrm{~mL})$ was collected in EDTA tubes (Becton Dickinson, Franklin Lakes, NJ, USA) and processed within $4 \mathrm{~h}$ from blood draw. Tubes were spun at $800 \mathrm{~g}$ for $15 \mathrm{~min}$ at room temperature. Plasma was then removed into new $15 \mathrm{~mL}$ tubes without disturbing the buffy coat and respun at $1600 \mathrm{~g}$ for 10 $\mathrm{min}$ at room temperature to remove cellular debris. Plasma was stored in $1-2 \mathrm{~mL}$ aliquots at $-80{ }^{\circ} \mathrm{C}$.

\subsection{Purification of Circulating Free (DNA) (cfDNA) from Plasma}

Plasma cfDNA was purified using the QIAamp circulating nucleic acid kit (Qiagen, Hilden, Germany) according to the manufacturer's instructions. cfDNA was purified from $4 \mathrm{~mL}$ of plasma except for Samples 13, 27, 69, 70, 76 and 80 for which 4.5-5 mL of plasma were used and Sample 34 for which $3.5 \mathrm{~mL}$ of plasma were used. The final elution volume was $25 \mu \mathrm{L}$ in all cases. cfDNA was subsequently quantified using a Qubit dsDNA high sensitivity assay kit and a Qubit fluorometer 3 (Life Technologies, Carlsbad, CA, USA), according to the manufacturer's instructions.

\subsection{Custom Melanoma Gene Panel for Targeted NGS of cfDNA}

An Ion Ampliseq HD made-to-order melanoma gene panel consisting of individual forward and reverse primers in 384 well-format to produce 123 amplicons (Table S1) was obtained from Life Technologies (Carlsbad, CA, USA). The panel covers 30 gene targets (Table S1 [30,47-53]) and was predicted to cover $90 \%$ of cutaneous melanoma (skin cutaneous melanoma TCGA dataset) and 95\% of uveal melanoma patient mutations (uveal melanoma TCGA dataset $[27,28]$ ). The panel covers nucleotide variants which give rise to melanoma-associated amino acid changes across 29 gene targets [47-52], as well as 6 melanoma-associated nucleotide variants in the promoter region of the TERT gene $[30,53]$.

In addition to running cfDNA from melanoma patients, analysis of the multiplex I cfDNA reference standard set (Cat\#HD780; Horizon Discovery, Waterbeach, UK) was also performed using the Ion Ampliseq HD custom panel. We generated a $0.26 \%$ MAF reference standard by diluting the provided 1.3\% MAF reference standard with the appropriate amount of the $100 \%$ wild-type reference standard. Details of library amplification and sequencing workflows for both the custom melanoma panel and an Oncomine pan cancer panel are provided in Supplementary Methods.

\section{4. ddPCR Analysis of ctDNA from Plasma}

The copy number of ctDNA per mL of plasma was determined using the QX200 droplet digital PCR (ddPCR) (Bio-Rad, Hercules, CA, USA) system to detect tumor-associated BRAF E586K, V600E/K, K601E, GNAQ R183C, Q209P or NRAS Q61K mutations, as previously described [8]. ddPCR mutation assays for BRAF E586K, K601E and GNAQ R183C were kindly provided by Elin Gray (Edith Cowan University) while the remainder were obtained from Bio-Rad (Hercules, CA, USA). TERT promoter mutations $-124 \mathrm{C}>\mathrm{T}$ and $-146 \mathrm{C}>\mathrm{T}$ were identified using ddPCR expert design assays dHsaEXD20945488 (TERT C228T_88) and dHsaEXD85215261 (TERT C250T_88) [54] (Bio-Rad, Hercules, CA, USA), according to the manufacturer's instructions. The TERT promoter assays were optimized by inclusion of $200 \mu \mathrm{M}$ 7-deaza-dGTP (New England Biolabs, Ipswich, MA, USA), as previously described [43]. The DNA copy number/mL of plasma for mutant and wild-type circulating DNA 
species was determined with Quantasoft software version 1.7.4 (Bio-Rad, Hercules, CA, USA) using a manual threshold setting. The minimum number of positive droplets for calling a mutation was set at two.

\subsection{Statistical Analysis}

One-way ANOVA with Geisser-Greenhouse correction and Tukey's multiple comparison test, comparing mean of each column with mean of every other column, were performed using Graphpad Prism version 8.3.1. Pearson correlation coefficient analysis, parametric paired $t$-tests and a Mann-Whitney unpaired, non-parametric $t$-test were also performed using Graphpad Prism version 8.3.1. Hierarchical clustering using Euclidean distance with complete linkage was undertaken using Morpheus (https://software.broadinstitute.org/morpheus). Venn diagrams were generated using the interactive online tool Venny 2.1 (http://bioinfogp.cnb.csic.es/tools/venny/index.html).

\section{Conclusions}

In this study, we developed a custom melanoma NGS panel. Analysis of a cohort of treatment-naïve melanoma patients revealed that sensitivity of ctDNA detection was influenced by the amount of cfDNA input and extracranial disease burden. With refinement, our panel will prove particularly useful in detecting tumor heterogeneity, potentially new targetable mutations and tracking treatment response and resistance in melanoma patients treated with systemic drug therapies. The development of a robust ctDNA profiling process will also reduce the need for invasive tissue biopsies and enable longitudinal, real-time monitoring of patients.

Supplementary Materials: The following are available online at http://www.mdpi.com/2072-6694/12/8/2228/s1. Supplementary methods; Figure S1: Summary amplicon performance; Table S1: Custom melanoma amplicons; Table S2: Summary total reads; Table S3: Summary matched patient samples; Table S4: Panel calibration; Table S5: Patient mutations identified; Table S6: Summary patient NGS; Table S7: Stage IV Concordance; Table S8: ddPCR vs. NGS; Table S9: Custom vs. pan; Table S10: TERT ddPCR.

Author Contributions: Conceptualization, R.J.D., J.H.L. and H.R.; methodology, R.J.D., J.H.L. and H.R.; formal analysis, R.J.D., J.H.L. and H.R.; investigation, R.J.D. and J.H.L.; resources, A.M.M., M.S.C., G.V.L., R.P.M.S., J.R.H., A.J.S., R.F.K. and R.A.S.; writing-original draft preparation, R.J.D. and H.R.; writing-review and editing, R.J.D., J.H.L., A.M.M., M.S.C., G.V.L., R.P.M.S., J.R.H., A.J.S., R.A.S., R.F.K. and H.R.; visualization, R.J.D., J.H.L. and H.R.; supervision, H.R.; project administration, H.R.; and funding acquisition, H.R. All authors have read and agreed to the published version of the manuscript.

Funding: R.J.D. was supported in part by a donation to Melanoma Institute Australia from the Clearbridge Foundation. This work was also supported in part by the National Health and Medical Research Council (APP1093017 and APP1128951). H.R., R.A.S. and G.V.L. are supported by National Health and Medical Research Council Research Fellowships (APP1104503, APP1141295 and APP1119059). G.V.L. is also supported by the Medical Foundation of The University of Sydney and Melanoma Institute Australia. A.M.M. is supported by a Cancer Institute NSW Fellowship and Melanoma Institute Australia. R.P.M.S. is supported by Melanoma Institute Australia. Support from colleagues at Melanoma Institute Australia, Royal Prince Alfred Hospital and Westmead Hospital is also gratefully acknowledged. Support from The Ainsworth Foundation is also gratefully acknowledged.

Acknowledgments: Technical support from Thermofisher personnel including Mike Payne, William Lo and Bo $\mathrm{Xu}$ is much appreciated.

Conflicts of Interest: G.V.L. receives consultant service fees from Amgen, BMS, Array, Pierre-Fabre, Novartis, Merck Sharp \& Dohme (MSD), Qbiotics, Roche and Sandoz. A.M.M. is an advisory board member for BMS, MSD, Novartis, Roche and Pierre Fabre. R.F.K. has been on advisory boards for Roche, Amgen, BMS, MSD, Novartis and TEVA and has received honoraria from MSD, BMS, Amgen and Novartis. M.S.C. is an advisory board member for MSD, BMS, Novartis, Pierre-Fabre, Roche and Amgen. R.A.S. has received fees for professional services from Qbiotics Group Limited, Novartis Pharma AG, MSD Sharp \& Dohme (Australia), NeraCare, AMGEN Inc., Bristol-Myers Squibb, Novartis Pharmaceuticals Australia Pty Limited, Myriad Genetics GmbH and GlaxoSmithKline Australia. R.P.M.S. has received honoraria for advisory board participation from MSD, Novartis and Qbiotics and speaking honoraria from BMS. These companies had no role in the design of the study; in the collection, analyses, or interpretation of data; in the writing of the manuscript, or in the decision to publish the results. All remaining authors have declared no conflicts of interest. 


\section{References}

1. De Rubis, G.; Krishnan, S.R.; Bebawy, M. Circulating tumor DNA-Current state of play and future perspectives. Pharmacol. Res. 2018, 136, 35-44. [CrossRef] [PubMed]

2. Donaldson, J.; Park, B.H. Circulating tumor DNA: Measurement and clinical utility. Ann. Rev. Med. 2018, 69, 223-234. [CrossRef] [PubMed]

3. Wan, J.C.M.; Massie, C.; Garcia-Corbacho, J.; Mouliere, F.; Brenton, J.D.; Caldas, C.; Pacey, S.; Baird, R.; Rosenfeld, N. Liquid biopsies come of age: Towards implementation of circulating tumour DNA. Nat. Rev. Cancer 2017, 17, 223-238. [CrossRef]

4. Yi, X.; Ma, J.; Guan, Y.; Chen, R.; Yang, L.; Xia, X. The feasibility of using mutation detection in ctDNA to assess tumor dynamics. Int. J. Cancer 2017, 140, 2642-2647. [CrossRef]

5. Scherer, F. Capturing tumor heterogeneity and clonal evolution by circulating tumor DNA profiling. Recent Results Cancer Res. 2020, 215, 213-230. [CrossRef] [PubMed]

6. Bronkhorst, A.J.; Ungerer, V.; Holdenrieder, S. The emerging role of cell-free DNA as a molecular marker for cancer management. Biomol. Detect. Quantif. 2019, 17, 100087. [CrossRef]

7. Gray, E.S.; Rizos, H.; Reid, A.L.; Boyd, S.C.; Pereira, M.R.; Lo, J.; Tembe, V.; Freeman, J.; Lee, J.H.; Scolyer, R.A.; et al. Circulating tumor DNA to monitor treatment response and detect acquired resistance in patients with metastatic melanoma. Oncotarget 2015, 6, 42008-42018. [CrossRef]

8. Lee, J.H.; Long, G.V.; Boyd, S.; Lo, S.; Menzies, A.M.; Tembe, V.; Guminski, A.; Jakrot, V.; Scolyer, R.A.; Mann, G.J.; et al. Circulating tumour DNA predicts response to anti-PD1 antibodies in metastatic melanoma. Ann. Oncol. 2017, 28, 1130-1136. [CrossRef]

9. Santiago-Walker, A.; Gagnon, R.; Mazumdar, J.; Casey, M.; Long, G.V.; Schadendorf, D.; Flaherty, K.; Kefford, R.; Hauschild, A.; Hwu, P.; et al. Correlation of BRAF mutation status in circulating-free DNA and tumor and association with clinical outcome across four BRAFi and MEKi clinical trials. Clin. Cancer Res. 2016, 22, 567-574. [CrossRef]

10. Valpione, S.; Gremel, G.; Mundra, P.; Middlehurst, P.; Galvani, E.; Girotti, M.R.; Lee, R.J.; Garner, G.; Dhomen, N.; Lorigan, P.C.; et al. Plasma total cell-free DNA (cfDNA) is a surrogate biomarker for tumour burden and a prognostic biomarker for survival in metastatic melanoma patients. Eur. J. Cancer 2018, 88, 1-9. [CrossRef]

11. Seremet, T.; Jansen, Y.; Planken, S.; Njimi, H.; Delaunoy, M.; El Housni, H.; Awada, G.; Schwarze, J.K.; Keyaerts, M.; Everaert, H.; et al. Undetectable circulating tumor DNA (ctDNA) levels correlate with favorable outcome in metastatic melanoma patients treated with anti-PD1 therapy. J. Transl. Med. 2019, 17, 303. [CrossRef] [PubMed]

12. Lee, J.H.; Saw, R.P.; Thompson, J.F.; Lo, S.; Spillane, A.J.; Shannon, K.F.; Stretch, J.R.; Howle, J.; Menzies, A.M.; Carlino, M.S.; et al. Pre-operative ctDNA predicts survival in high-risk stage III cutaneous melanoma patients. Ann. Oncol. 2019, 30, 815-822. [CrossRef] [PubMed]

13. Lee, R.J.; Gremel, G.; Marshall, A.; Myers, K.A.; Fisher, N.; Dunn, J.A.; Dhomen, N.; Corrie, P.G.; Middleton, M.R.; Lorigan, P.; et al. Circulating tumor DNA predicts survival in patients with resected high-risk stage II/III melanoma. Ann. Oncol. 2018, 29, 490-496. [CrossRef] [PubMed]

14. McEvoy, A.C.; Pereira, M.R.; Reid, A.; Pearce, R.; Cowell, L.; Al-Ogaili, Z.; Khattak, M.A.; Millward, M.; Meniawy, T.M.; Gray, E.S.; et al. Monitoring melanoma recurrence with circulating tumor DNA: A proof of concept from three case studies. Oncotarget 2019, 10, 113-122. [CrossRef] [PubMed]

15. Tan, L.; Sandhu, S.; Lee, R.J.; Li, J.; Callahan, J.; Ftouni, S.; Dhomen, N.; Middlehurst, P.; Wallace, A.; Raleigh, J.; et al. Prediction and monitoring of relapse in stage III melanoma using circulating tumor DNA. Ann. Oncol. 2019, 30, 804-814. [CrossRef] [PubMed]

16. Diefenbach, R.J.; Lee, J.H.; Strbenac, D.; Yang, J.Y.H.; Menzies, A.M.; Carlino, M.S.; Long, G.V.; Spillane, A.J.; Stretch, J.R.; Saw, R.P.M.; et al. Analysis of the whole-exome sequencing of tumor and circulating tumor DNA in metastatic melanoma. Cancers (Basel) 2019, 11, 1905. [CrossRef]

17. McEvoy, A.C.; Warburton, L.; Al-Ogaili, Z.; Celliers, L.; Calapre, L.; Pereira, M.R.; Khattak, M.A.; Meniawy, T.M.; Millward, M.; Ziman, M.; et al. Correlation between circulating tumour DNA and metabolic tumour burden in metastatic melanoma patients. BMC Cancer 2018, 18, 726. [CrossRef] 
18. Lee, J.H.; Long, G.V.; Menzies, A.M.; Lo, S.; Guminski, A.; Whitbourne, K.; Peranec, M.; Scolyer, R.; Kefford, R.F.; Rizos, H.; et al. Association between circulating tumor DNA and pseudoprogression in patients with metastatic melanoma treated with anti-programmed cell death 1 antibodies. JAMA Oncol. 2018, 4, 717-721. [CrossRef]

19. Sanmamed, M.F.; Fernandez-Landazuri, S.; Rodriguez, C.; Zarate, R.; Lozano, M.D.; Zubiri, L.; Perez-Gracia, J.L.; Martin-Algarra, S.; Gonzalez, A. Quantitative cell-free circulating BRAFV600E mutation analysis by use of droplet digital PCR in the follow-up of patients with melanoma being treated with BRAF inhibitors. Clin. Chem. 2015, 61, 297-304. [CrossRef]

20. Bai, Y.; Wang, Z.; Liu, Z.; Liang, G.; Gu, W.; Ge, Q. Technical progress in circulating tumor DNA analysis using next generation sequencing. Mol. Cell. Probes 2020, 49, 101480. [CrossRef]

21. Zhou, Q.; Moser, T.; Perakis, S.; Heitzer, E. Untargeted profiling of cell-free circulating DNA. Transl. Cancer Res. 2018, 7, S140-S152. [CrossRef]

22. Diefenbach, R.J.; Lee, J.H.; Rizos, H. Monitoring melanoma using circulating free DNA. Am. J. Clin. Dermatol. 2019, 20, 1-12. [CrossRef] [PubMed]

23. Gorges, K.; Wiltfang, L.; Gorges, T.M.; Sartori, A.; Hildebrandt, L.; Keller, L.; Volkmer, B.; Peine, S.; Babayan, A.; Moll, I.; et al. Intra-patient heterogeneity of circulating tumor cells and circulating tumor DNA in blood of melanoma patients. Cancers (Basel) 2019, 11, 1685. [CrossRef] [PubMed]

24. Gray, E.S.; Witkowski, T.; Pereira, M.; Calapre, L.; Herron, K.; Irwin, D.; Chapman, B.; Khattak, M.A.; Raleigh, J.; Hatzimihalis, A.; et al. Genomic Analysis of Circulating Tumor DNA Using a Melanoma-Specific UltraSEEK Oncogene Panel. J. Mol. Diagn. 2019, 21, 418-426. [CrossRef]

25. Schneegans, S.; Luck, L.; Besler, K.; Bluhm, L.; Stadler, J.C.; Staub, J.; Greinert, R.; Volkmer, B.; Kubista, M.; Gebhardt, C.; et al. Pre-analytical factors affecting the establishment of a single tube assay for multiparameter liquid biopsy detection in melanoma patients. Mol. Oncol. 2020, 14, 1001-1015. [CrossRef]

26. Calapre, L.; Giardina, T.; Robinson, C.; Reid, A.L.; Al-Ogaili, Z.; Pereira, M.R.; McEvoy, A.C.; Warburton, L.; Hayward, N.K.; Khattak, M.A.; et al. Locus-specific concordance of genomic alterations between tissue and plasma circulating tumor DNA in metastatic melanoma. Mol. Oncol. 2019, 13, 171-184. [CrossRef]

27. Cerami, E.; Gao, J.; Dogrusoz, U.; Gross, B.E.; Sumer, S.O.; Aksoy, B.A.; Jacobsen, A.; Byrne, C.J.; Heuer, M.L.; Larsson, E.; et al. The cBio cancer genomics portal: An open platform for exploring multidimensional cancer genomics data. Cancer Discov. 2012, 2, 401-404. [CrossRef]

28. Gao, J.; Aksoy, B.A.; Dogrusoz, U.; Dresdner, G.; Gross, B.; Sumer, S.O.; Sun, Y.; Jacobsen, A.; Sinha, R.; Larsson, E.; et al. Integrative analysis of complex cancer genomics and clinical profiles using the cBioPortal. Sci. Signal. 2013, 6, 11. [CrossRef]

29. Nagore, E.; Heidenreich, B.; Rachakonda, S.; Garcia-Casado, Z.; Requena, C.; Soriano, V.; Frank, C.; Traves, V.; Quecedo, E.; Sanjuan-Gimenez, J.; et al. TERT promoter mutations in melanoma survival. Int. J. Cancer 2016, 139, 75-84. [CrossRef]

30. Heidenreich, B.; Nagore, E.; Rachakonda, P.S.; Garcia-Casado, Z.; Requena, C.; Traves, V.; Becker, J.; Soufir, N.; Hemminki, K.; Kumar, R. Telomerase reverse transcriptase promoter mutations in primary cutaneous melanoma. Nat. Commun. 2014, 5, 3401. [CrossRef]

31. Etemadmoghadam, D.; Azar, W.J.; Lei, Y.; Moujaber, T.; Garsed, D.W.; Kennedy, C.J.; Fereday, S.; Mitchell, C.; Chiew, Y.E.; Hendley, J.; et al. EIF1AX and NRAS mutations co-occur and cooperate in low-grade serous ovarian carcinomas. Cancer Res. 2017, 77, 4268-4278. [CrossRef] [PubMed]

32. Rose, A.M.; Luo, R.; Radia, U.K.; Kalirai, H.; Thornton, S.; Luthert, P.J.; Jayasena, C.N.; Verity, D.H.; Coupland, S.E.; Rose, G.E. Detection of mutations in SF3B1, EIF1AX and GNAQ in primary orbital melanoma by candidate gene analysis. BMC Cancer 2018, 18, 1262. [CrossRef] [PubMed]

33. Phung, B.; Ciesla, M.; Sanna, A.; Guzzi, N.; Beneventi, G.; Ngoc, P.C.T.; Lauss, M.; Cabrita, R.; Cordero, E.; Bosch, A.; et al. The X-Linked DDX3X RNA helicase dictates translation reprogramming and metastasis in melanoma. Cell Rep. 2019, 27, 3573-3586.e7. [CrossRef] [PubMed]

34. Warton, K.; Lin, V.; Navin, T.; Armstrong, N.J.; Kaplan, W.; Ying, K.; Gloss, B.; Mangs, H.; Nair, S.S.; Hacker, N.F.; et al. Methylation-capture and next-generation sequencing of free circulating DNA from human plasma. BMC Genom. 2014, 15, 476. [CrossRef]

35. Chen, S.; Wang, Q.; Yu, H.; Capitano, M.L.; Vemula, S.; Nabinger, S.C.; Gao, R.; Yao, C.; Kobayashi, M.; Geng, Z.; et al. Mutant p53 drives clonal hematopoiesis through modulating epigenetic pathway. Nat. Commun. 2019, 10, 5649. [CrossRef] [PubMed] 
36. Genovese, G.; Kahler, A.K.; Handsaker, R.E.; Lindberg, J.; Rose, S.A.; Bakhoum, S.F.; Chambert, K.; Mick, E.; Neale, B.M.; Fromer, M.; et al. Clonal hematopoiesis and blood-cancer risk inferred from blood DNA sequence. N. Engl. J. Med. 2014, 371, 2477-2487. [CrossRef]

37. Jaiswal, S.; Fontanillas, P.; Flannick, J.; Manning, A.; Grauman, P.V.; Mar, B.G.; Lindsley, R.C.; Mermel, C.H.; Burtt, N.; Chavez, A.; et al. Age-related clonal hematopoiesis associated with adverse outcomes. N. Engl. J. Med. 2014, 371, 2488-2498. [CrossRef]

38. Xie, M.; Lu, C.; Wang, J.; McLellan, M.D.; Johnson, K.J.; Wendl, M.C.; McMichael, J.F.; Schmidt, H.K.; Yellapantula, V.; Miller, C.A.; et al. Age-related mutations associated with clonal hematopoietic expansion and malignancies. Nat. Med. 2014, 20, 1472-1478. [CrossRef]

39. McEvoy, A.C.; Calapre, L.; Pereira, M.R.; Giardina, T.; Robinson, C.; Khattak, M.A.; Meniawy, T.M.; Pritchard, A.L.; Hayward, N.K.; Amanuel, B.; et al. Sensitive droplet digital PCR method for detection of TERT promoter mutations in cell free DNA from patients with metastatic melanoma. Oncotarget 2017, 8, 78890-78900. [CrossRef]

40. Chang, G.A.; Wiggins, J.M.; Corless, B.C.; Syeda, M.M.; Tadepalli, J.S.; Blake, S.; Fleming, N.; Darvishian, F.; Pavlick, A.; Berman, R.; et al. TERT, BRAF, and NRAS mutational heterogeneity between paired primary and metastatic melanoma tumors. J. Invest. Dermatol. 2020. [CrossRef]

41. Shaib, W.L.; Zakka, K.; Staley, C., III; Roberts, A.; Akce, M.; Wu, C.; Alese, O.B.; El-Rayes, B.F. Blood-based next-generation sequencing analysis of appendiceal cancers. Oncologist 2019. [CrossRef]

42. Mody, K.; Kasi, P.M.; Yang, J.D.; Surapaneni, P.K.; Ritter, A.; Roberts, A.; Nagy, R.; Borad, M.J. Feasibility of circulating tumor DNA testing in hepatocellular carcinoma. J. Gastrointest Oncol. 2019, 10, 745-750. [CrossRef] [PubMed]

43. Colebatch, A.J.; Witkowski, T.; Waring, P.M.; McArthur, G.A.; Wong, S.Q.; Dobrovic, A. Optimizing amplification of the GC-rich TERT promoter region using 7-deaza-dGTP for droplet digital PCR quantification of TERT promoter mutations. Clin. Chem. 2018, 64, 745-747. [CrossRef] [PubMed]

44. Kriegsmann, M.; Arens, N.; Endris, V.; Weichert, W.; Kriegsmann, J. Detection of KRAS, NRAS and BRAF by mass spectrometry-A sensitive, reliable, fast and cost-effective technique. Diagn. Pathol. 2015, 10, 132. [CrossRef] [PubMed]

45. Giannini, R.; Lupi, C.; Sensi, E.; Ali, G.; Proietti, A.; Boldrini, L.; Servadio, A.; Giordano, M.; Macerola, E.; Bruno, R.; et al. EGFR and KRAS mutational analysis in a large series of Italian non-small cell lung cancer patients: 2,387 cases from a single center. Oncol. Rep. 2016, 36, 1166-1172. [CrossRef]

46. Kakavand, H.; Walker, E.; Lum, T.; Wilmott, J.S.; Selinger, C.I.; Smith, E.; Saw, R.P.; Yu, B.; Cooper, W.A.; Long, G.V.; et al. BRAF(V600E) and NRAS(Q61L/Q61R) mutation analysis in metastatic melanoma using immunohistochemistry: A study of 754 cases highlighting potential pitfalls and guidelines for interpretation and reporting. Histopathology 2016, 69, 680-686. [CrossRef]

47. Robertson, A.G.; Shih, J.; Yau, C.; Gibb, E.A.; Oba, J.; Mungall, K.L.; Hess, J.M.; Uzunangelov, V.; Walter, V.; Danilova, L.; et al. Integrative analysis identifies four molecular and clinical subsets in uveal melanoma. Cancer Cell 2017, 32, 204-220.e15. [CrossRef]

48. Hodis, E.; Watson, I.R.; Kryukov, G.V.; Arold, S.T.; Imielinski, M.; Theurillat, J.P.; Nickerson, E.; Auclair, D.; Li, L.; Place, C.; et al. A landscape of driver mutations in melanoma. Cell 2012, 150, 251-263. [CrossRef]

49. Arafeh, R.; Qutob, N.; Emmanuel, R.; Keren-Paz, A.; Madore, J.; Elkahloun, A.; Wilmott, J.S.; Gartner, J.J.; Di Pizio, A.; Winograd-Katz, S.; et al. Recurrent inactivating RASA2 mutations in melanoma. Nat. Genet. 2015, 47, 1408-1410. [CrossRef]

50. Akbani, R.; Akdemir, K.C.; Aksoy, B.A.; Albert, M.; Ally, A.; Amin, S.B.; Arachchi, H.; Arora, A.; Auman, J.T.; Ayala, B.; et al. Genomic classification of cutaneous melanoma. Cell 2015, 161, 1681-1696. [CrossRef]

51. Bailey, M.H.; Tokheim, C.; Porta-Pardo, E.; Sengupta, S.; Bertrand, D.; Weerasinghe, A.; Colaprico, A.; Wendl, M.C.; Kim, J.; Reardon, B.; et al. Comprehensive characterization of cancer driver genes and mutations. Cell 2018, 173, 371-385.e18. [CrossRef] [PubMed]

52. Hayward, N.K.; Wilmott, J.S.; Waddell, N.; Johansson, P.A.; Field, M.A.; Nones, K.; Patch, A.M.; Kakavand, H.; Alexandrov, L.B.; Burke, H.; et al. Whole-genome landscapes of major melanoma subtypes. Nature 2017, 545, 175-180. [CrossRef] [PubMed] 
53. Horn, S.; Figl, A.; Rachakonda, P.S.; Fischer, C.; Sucker, A.; Gast, A.; Kadel, S.; Moll, I.; Nagore, E.; Hemminki, K.; et al. TERT promoter mutations in familial and sporadic melanoma. Science 2013, 339, 959-961. [CrossRef] [PubMed]

54. Corless, B.C.; Chang, G.A.; Cooper, S.; Syeda, M.M.; Shao, Y.; Osman, I.; Karlin-Neumann, G.; Polsky, D. Development of novel mutation-specific droplet digital PCR assays detecting TERT promoter mutations in tumor and plasma samples. J. Mol. Diagn 2019, 21, 274-285. [CrossRef] [PubMed]

(c) (2)

(C) 2020 by the authors. Licensee MDPI, Basel, Switzerland. This article is an open access article distributed under the terms and conditions of the Creative Commons Attribution (CC BY) license (http://creativecommons.org/licenses/by/4.0/). 